\title{
Efficiency enhancement by reconfigurable matching networks in LINC transmitters
}

\author{
Carlos Mateo* (D, Pedro L. Carro, Paloma Garcia-Ducar, Jesus de Mingo, Jose Ramon Perez-Cisneros \\ and Cesar Sanchez-Perez
}

\begin{abstract}
This paper proposes the use of a transmitter based on a linear amplification with nonlinear components (LINC) architecture, in which the reconfigurable matching networks (RMNs) are included. By varying the RMN active cell number, it is possible to change the load impedance at the power amplifier (PA) output, improving the amplifier drain efficiency and therefore the efficiency of the whole system. A long-term evolution (LTE) downlink signal with a bandwidth of $1.4 \mathrm{MHz}$ and a peak-to-average power ratio (PAPR) of $11.48 \mathrm{~dB}$ is applied in order to carry out the experiments. Results show that the use of the RMNs in a LINC architecture improves the efficiency at all tested frequencies, especially at $927 \mathrm{MHz}$ reaching an enhancement of 36.50\%. Regarding the distortion, the adjacent channel leakage ratio (ACLR) values increase in all cases, with an improvement of $3.5 \mathrm{~dB}$ at $958 \mathrm{MHz}$. Finally, in terms of error vector magnitude (EVM), the proposed architecture offers a value of $1.96 \%$ at $927 \mathrm{MHz}$.
\end{abstract}

Keywords: Drain efficiency, Linear amplification with nonlinear components (LINC), Reconfigurable matching network (RMN), Power amplifier

\section{Introduction}

The efficiency-linearity trade-off is a very challenging aspect in the design of radiofrequency (RF) front-ends in modern communication systems [1, 2]. Many techniques have been proposed to improve the efficiency of the power stages in the wireless RF front-ends, such as Doherty amplifiers [3], envelope tracking [4], or linear amplification with nonlinear components (LINC) [5-7]. The first one uses an active output impedance load-pulling, increasing the efficiency without compromising the maximum output power. The envelope tracking technique may enable the RF power amplifier (PA) to be operated continuously in the compression region, obtaining an improvement in the power efficiency. The LINC technique can achieve linear amplification while using very nonlinear and power-efficient amplifiers by means of two-phase modulated signals with a constant envelope.

Reconfigurable matching networks (RMNs) are used for improving PA efficiency and frequency reconfigurability

\footnotetext{
*Correspondence: cmperez@unizar.es

Department of Electronic Engineering and Communications, Aragon Institute of Engineering Research (I3A), University of Zaragoza, Mariano Esquillor, 50018
} Zaragoza, Spain and changing load impedance. This is the approach of dynamic load modulation (DLM) [8]. Recently, some papers have been published using varactor-based matching networks. They offer very promising outcomes in transmitter efficiency enhancements [9]. In this work, we propose the use of a transmitter based on a LINC architecture, in which the RMNs are included to improve the efficiency. The setup uses two RMNs which are placed between the PA output and the combiner input so as to enhance the PA efficiency and, therefore, the efficiency of the whole system.

This paper is organized as follows. Section 2 presents the LINC architecture as well as the fundamentals of RMNs and their integration in the system. Experimental results are shown in Section 3, and finally, conclusions are stated in Section 4.

\section{RMN-LINC architecture}

The classical LINC amplification scheme decomposes a varying envelope signal $s(t)$ into two constant envelope signals.

$$
s(t)=r(t) \cdot e^{j \phi(t)}=s_{1}(t)+s_{2}(t) .
$$

\section{Springer Open}

(c) The Author(s). 2017 Open Access This article is distributed under the terms of the Creative Commons Attribution 4.0 International License (http://creativecommons.org/licenses/by/4.0/), which permits unrestricted use, distribution, and reproduction in any medium, provided you give appropriate credit to the original author(s) and the source, provide a link to the Creative Commons license, and indicate if changes were made. 
These signals $s_{1}(t)$ and $s_{2}(t)$ can be obtained according to the following equation

$$
\left\{\begin{array}{l}
s_{1}(t)=0.5 \cdot s(t)[1+j \cdot e(t)] \\
s_{2}(t)=0.5 \cdot s(t)[1-j \cdot e(t)]
\end{array}\right.
$$

where $e(t)$ is given by $e(t)=\sqrt{\left(r_{\max }^{2} / r(t)^{2}\right)-1}$ and $r_{\text {max }}$ and $r(t)$ represent the maximum and instantaneous $s(t)$ magnitude values, respectively.

The efficiency strongly depends on the envelope signal probability density function (PDF), but LINC waveforms are constant envelope signals, simplifying considerably the efficiency analysis (drain efficiency and averaged drain efficiency agree). The impact of the isolated combiner on the overall transmitter efficiency has not been taken into account in this work, but its main effects are summarized in [10].

The PA drain and combiner efficiencies are defined as

$$
\eta_{\mathrm{PA}}^{(m)}=\frac{P_{\mathrm{RF}}^{(m)}}{P_{\mathrm{DC}}^{(m)}}=\frac{P_{\mathrm{RF}}^{(m)}}{I_{\mathrm{DD}}^{(m)} \cdot V_{\mathrm{DD}}} \quad \eta_{\mathrm{comb}}=\frac{P_{\mathrm{RF}_{\text {out }}}}{P_{\mathrm{RF}}^{(1)}+P_{\mathrm{RF}}^{(2)}},
$$

with $m=1,2$ the paths, $V_{D D}$ and $I_{D D}$ are the voltage and current consumption by the PA, respectively, and the $P_{R F}^{(m)}$ is the output power. $P_{R F_{\text {out }}}$ is the combiner output power, and $P_{R F}^{(1)}$ and $P_{R F}^{(2)}$ are the input power in both branches. From (3), the efficiency of the whole system is

$$
\eta_{\mathrm{LINC}}=\frac{P_{\mathrm{RF}_{\mathrm{out}}}}{P_{\mathrm{DC}}^{(1)}+P_{\mathrm{DC}}^{(2)}}=\eta_{\mathrm{comb}} \cdot \frac{P_{\mathrm{RF}}^{(1)}+P_{\mathrm{RF}}^{(2)}}{P_{\mathrm{DC}}^{(1)}+P_{\mathrm{DC}}^{(2)}} .
$$

According to [11], there is an optimum load impedance that maximizes efficiency for each frequency. A wideband PA, for example, cannot guarantee for each frequency that the optimum load pull impedance is presented to the transistor drain, and consequently, some efficiency is lost in such designs. If the RMN is added to the classical LINC structure (see Fig. 1), the transistor can be theoretically accurately loaded, which increases PA efficiency.

In order to get some insight about how the RMNs may achieve this impedance, we model the RMNs and the combiner as linear networks so that the S-parameter approach can be valid. Additional simplifications stem from the fact that the combiner is matched to the antenna to $Z_{0}$; although if this condition was not fulfilled, the following analysis would be still valid. Each RMN is modeled by an S-parameter network which can electronically be tuned depending on the RMN design, namely discrete (with switches for example) or continuous (mainly with varactors). In this work, we focus on discrete RMNs because it is easier to control the network by means of digital platforms based on field programmable gate array (FPGAs) for example, and control algorithms are out of the scope of the article.

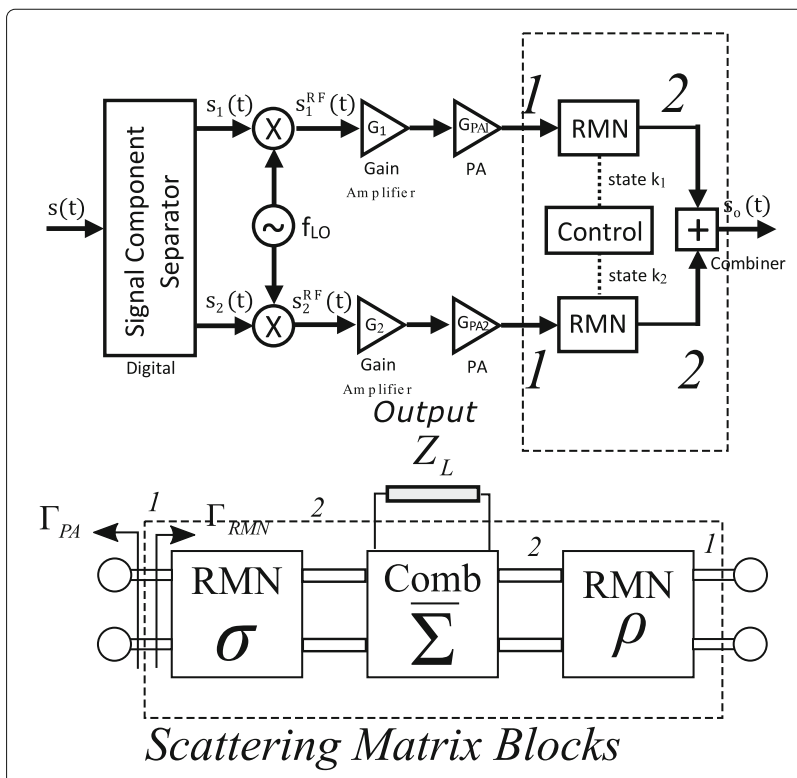

Fig. 1 Proposed RMN-LINC architecture and simplified RMN box model

To compute the input and output reflection coefficients presented to each PA at certain states $k_{i}$ and $k_{q}$ and frequency, the RMNs in each branch are modeled with the S-parameter matrices $\sigma$ and $\rho$ as

$$
\begin{aligned}
& \mathrm{S}_{\mathrm{RMN} 1}=\sigma\left(k_{i}\right)=\left(\begin{array}{cc}
\sigma_{11}\left(k_{i}\right) & \sigma_{12}\left(k_{i}\right) \\
\sigma_{21}\left(k_{i}\right) & \sigma_{22}\left(k_{i}\right)
\end{array}\right) \\
& \mathrm{S}_{\mathrm{RMN} 2}=\rho\left(k_{q}\right)=\left(\begin{array}{ll}
\rho_{11}\left(k_{q}\right) & \rho_{12}\left(k_{q}\right) \\
\rho_{21}\left(k_{q}\right) & \rho_{22}\left(k_{q}\right)
\end{array}\right) .
\end{aligned}
$$

Although the combiner is a three-port device, it is perfectly described by means of two-port matrix once the third port is properly loaded. If it is symmetrical and reciprocal

$$
\mathrm{S}_{\mathrm{comb}}=\left(\begin{array}{cc}
\alpha & \delta \\
\delta & \alpha
\end{array}\right)
$$

where $\alpha$ are the reflection coefficients in the ports connected to the RMNs and $\delta$ models their isolation. In an isolated matched combiner $\alpha \approx 0$ and $\delta \approx 0$. Combining the two networks using $S$-parameters $S^{(A)}$ and $S^{(B)}$,

$$
S^{A, B}=\left(\begin{array}{cc}
S_{11}^{(A)}+\frac{S_{12}^{(A)} S_{21}^{(A)} S_{11}^{(B)}}{1-S_{22}^{A} S_{11}^{B}} & \frac{S_{12}^{(A)} S_{12}^{(B)}}{1-S_{22}^{A} S_{11}^{B}} \\
\frac{S_{21}^{(A)} S_{21}^{(B)}}{1-S_{22}^{A} S_{11}^{B}} & \frac{S_{12}^{(B)} S_{21}^{(B)} S_{22}^{(A)}}{1-S_{22}^{A} S_{11}^{B}}+S_{22}^{(B)}
\end{array}\right)
$$

the total scattering matrix corresponding to a RMN with a fixed state (in the following, the index $k$ is omitted due to simplicity reasons) and the combiner is

$$
\mathrm{S}_{\mathrm{RMN}-\mathrm{COMB}}=\left(\begin{array}{cc}
\sigma_{11}+\frac{\sigma_{12} \sigma_{21}}{1-\sigma_{22} \alpha} & \frac{\sigma_{12} \delta}{1-\sigma_{22} \alpha} \\
\frac{\sigma_{21} \delta}{1-\sigma_{22} \alpha} & \frac{\delta^{2} \sigma_{22}}{1-\sigma_{22} \alpha}+\alpha
\end{array}\right) .
$$




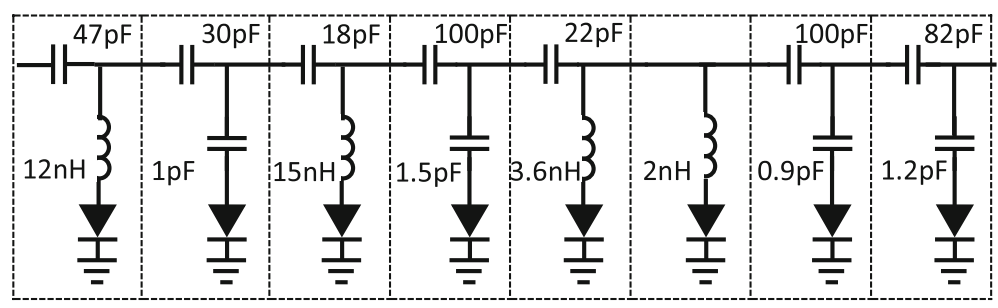

Fig. 2 Eight-cell RMN design schematic

Finally, the $S_{11}$ of whole cascade is computed

$$
\mathrm{S}_{11}^{\mathrm{TOTAL}}=\sigma_{11}+\frac{\sigma_{12} \sigma_{21} \alpha}{1-\sigma_{22} \alpha}+\frac{\frac{\sigma_{12} \delta}{1-\sigma_{22} \alpha} \frac{\sigma_{21} \delta}{1-\sigma_{22} \alpha} \rho_{22}}{1-\left(\frac{\delta^{2} \sigma_{22}}{1-\sigma_{22} \alpha}+\alpha\right) \rho_{22}} .
$$

If the RMNs are reciprocal, which is usually fulfilled, $\sigma_{12}=\sigma_{21}$, and after some algebraic simplifications,

$$
\mathrm{S}_{11}^{\mathrm{TOTAL}}=\sigma_{11}+\frac{\sigma^{2} \alpha}{1-\sigma_{22} \alpha}+F(\delta)
$$

where $F(\delta)$ is a factor which depends on the combiner isolation,

$$
F(\delta)=\frac{\sigma^{2} \delta^{2} \rho_{22}}{\left(1-\sigma_{22} \alpha\right)^{2}-\rho_{22} \delta^{2} \sigma_{22}\left(1-\sigma_{22} \alpha\right)+\rho_{22}\left(1-\sigma_{22}\right)^{2}} .
$$

At this point, let us notice that $\delta^{2}$ is really small, as $\delta \approx 0$. Therefore, (10) can be safely approximated as

$$
\mathrm{S}_{11}^{\mathrm{TOTAL}} \simeq \sigma_{11}+\frac{\sigma^{2} \alpha}{1-\sigma_{22} \alpha}
$$

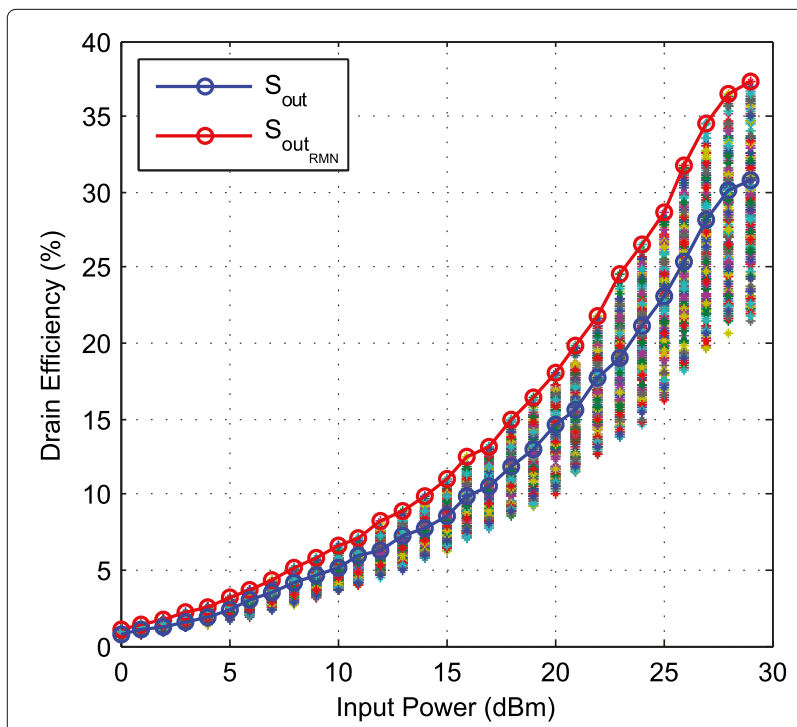

Fig. 3 Averaged drain efficiency curves for all RMN states, for the PA with and without the RMN, at frequency of $958 \mathrm{MHz}$ in the path 1 which is equivalent to say that both branches are nearly independent. This is important because it allows the RMN input impedance not only to be calculated neglecting the PA load in the other branch but also to individually perform an approximation to the optimum state in each branch. In terms of matching domain and recovering the state index $k_{i}$, the capability of the RMN approach is summarized in

$$
Z_{\mathrm{RMN}}=Z_{0} \frac{1+S_{11}\left(k_{i}\right)}{1-S_{11}\left(k_{i}\right)}=Z_{0} \frac{1+\sigma_{11}\left(k_{i}\right)+\frac{\sigma^{2}\left(k_{i}\right) \alpha}{1-\sigma_{22}\left(k_{i}\right) \alpha}}{1-\sigma_{11}\left(k_{i}\right)-\frac{\sigma^{2}\left(k_{i}\right) \alpha}{1-\sigma_{22}\left(k_{i}\right) \alpha}} .
$$

Simplifying,

$$
Z_{\mathrm{RMN}}\left(k_{i}\right)=Z_{0} \frac{1+\sigma_{11}\left(k_{i}\right)+\alpha P\left(\mathrm{~S}_{R M N 1}\left(k_{i}\right)\right)}{1-\sigma_{11}\left(k_{i}\right)-\alpha Q\left(\mathrm{~S}_{R M N 1}\left(k_{i}\right)\right)},
$$

where $P(\cdot), Q(\cdot)$ are functions of the $S$-parameter matrix elements. Equation (14) suggests that the matching domain does not depend simply on the RMN but also on the combiner. However, this dependence should be a

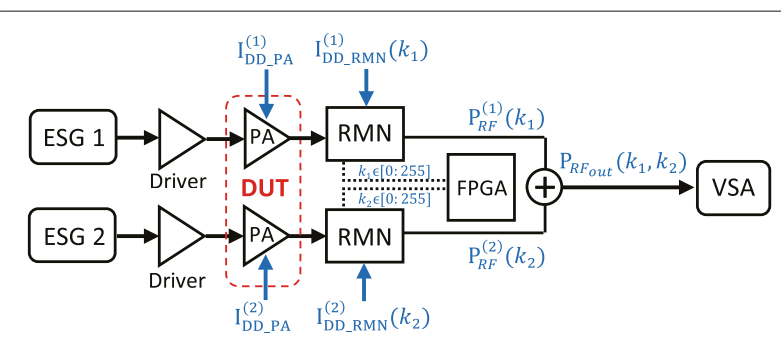

(a)

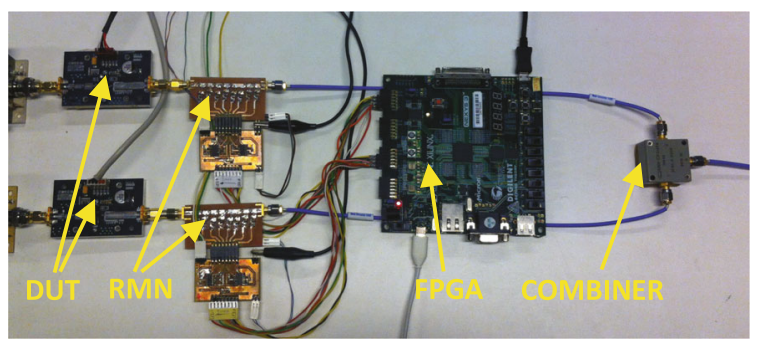

(b)

Fig. 4 Experimental setup with the RMNs (a) and the details (b) 
Table 1 RMN performance at different frequencies

\begin{tabular}{|c|c|c|c|c|c|c|c|c|c|c|}
\hline $\begin{array}{l}\text { Freq } \\
(\mathrm{MHz})\end{array}$ & $\begin{array}{l}\mathrm{RMN}_{1} \\
\text { (state) }\end{array}$ & $\begin{array}{l}\mathrm{RMN}_{2} \\
\text { (state) }\end{array}$ & $\begin{array}{l}\mathrm{Pdc}_{2} \\
(\mathrm{~W})\end{array}$ & $\begin{array}{l}\mathrm{Pdc}_{2} \\
(\mathrm{~W})\end{array}$ & $\begin{array}{l}\mathrm{GT}_{1}(\min , \max ) \\
(\mathrm{dB})\end{array}$ & $\begin{array}{l}\mathrm{GT}_{2}(\min , \max ) \\
(\mathrm{dB})\end{array}$ & $\begin{array}{l}\mathrm{L}_{\Omega} 1 \\
(\mathrm{~dB})\end{array}$ & $\begin{array}{l}L_{\Omega-2} \\
(d B)\end{array}$ & $\begin{array}{l}\mathrm{IL}_{1} \\
(\mathrm{~dB})\end{array}$ & $\begin{array}{l}\mathrm{IL}_{2} \\
(\mathrm{~dB})\end{array}$ \\
\hline 927 & 163 & 203 & 0.95 & 1.14 & {$[-12.11,-0.49]$} & {$[-9.81,-0.92]$} & 0.47 & 0.78 & 7.25 & 5.08 \\
\hline 942 & 35 & 163 & 0.76 & 0.95 & {$[-10.84,-0.46]$} & {$[-12.24,-0.55]$} & 0.44 & 0.53 & 6.24 & 7.28 \\
\hline 958 & 167 & 219 & 1.14 & 1.33 & {$[-12.72,-0.84]$} & {$[-9.44,-0.73]$} & 0.70 & 0.67 & 5.26 & 5.10 \\
\hline
\end{tabular}

second-order effect, as the combiner is supposed to be well matched.

Figure 2 shows the RMN circuit which is used in this work and whose design details are described in [12]. It consists of an eight-cell structure composed by inductors and capacitors. There is a PIN diode in each cell which is used as a switch element $S W i$, with $i=1, \ldots, 8$. A bias circuit controls every switch avoiding RF signal leakage and blocking DC bias. A FPGA supplies $\pm 5 V$ to each cell depending on the RMN state. Thus, the total RMN consumption is the sum of every individual active cell consumption. The RMN is a discrete topology, and it is a possible candidate for the RMN-LINC architecture, but a continuous RMN based on varactors could also be feasible. Figure 3 shows the improvement in drain efficiency that this circuit provides depending on the RMN state.

The RMN-LINC architecture can achieve higher efficiencies either because of a higher RF output power (keeping power consumption) or a reduction in DC power with the same RF output power. Both factors depend on the selected RMN state. However, the RMN requires its own power to operate (the greater the number of active switches, the larger the RMN consumption).

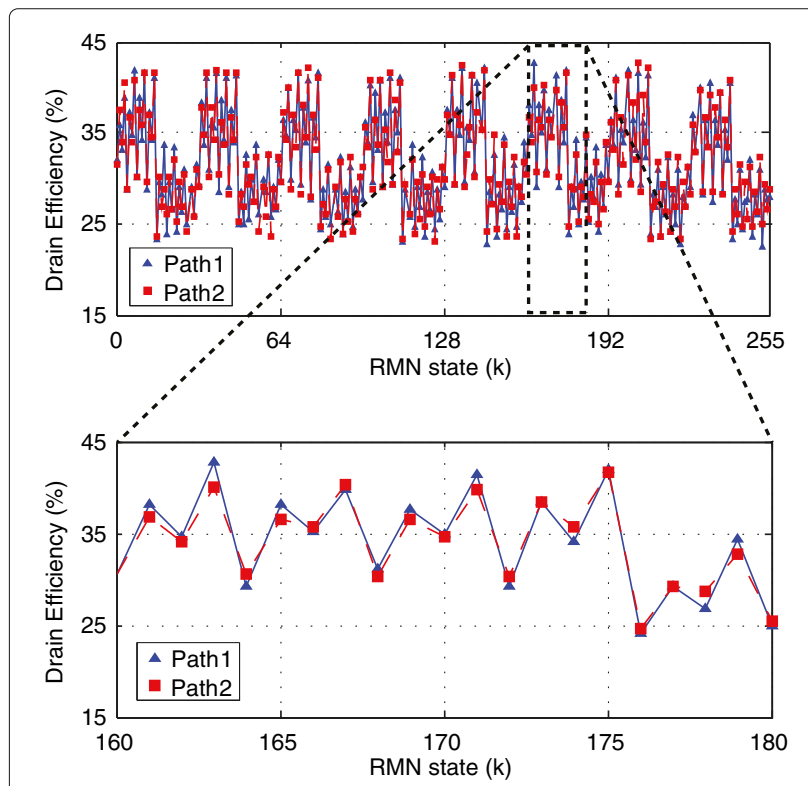

Fig. 5 Averaged drain efficiency measured in both paths with the RMNs in the setup
Thus, total efficiency (PA $+\mathrm{RMN})$ is a function of the state $\left(k_{m}\right)$

$$
\overline{\eta_{\mathrm{PA}+\mathrm{RMN}}^{(m)}}=\frac{\overline{P_{\mathrm{RF}}^{(m)}\left(k_{m}\right)}}{\overline{P_{\mathrm{DC}}^{(m)}\left(k_{m}\right)}}=\frac{\overline{P_{\mathrm{RF}}^{(m)}\left(k_{m}\right)}}{\overline{P_{\mathrm{DC} \_ \text {PA }}^{(m)}\left(k_{m}\right)}+\overline{P_{\mathrm{DC} \_\mathrm{RMN}}^{(m)}\left(k_{m}\right)}},
$$

with $m=1,2$ the paths, $k_{m} \in[0,255]$ the RMN state, and $P_{D C_{-} P A}$ and $P_{D C_{-} R M N}$ the DC consumption of the PA and the RMN, respectively. Without loss of generality, the $k_{m}$ coefficient is omitted to simplify equations. The efficiency of the whole system is

$$
\eta_{\text {LINC_RMN }}=\eta_{c o m b} \cdot \frac{\sum_{m=1}^{2} P_{R F}^{(m)}}{\sum_{m=1}^{2} P_{D C_{-} P A}^{(m)}+P_{D C_{-} R M N}^{(m)}}
$$

\section{Experimental results and discussion}

\subsection{Proposed setup}

The proposed test setup schematic is shown in Fig. 4a. The signals $s_{1}$ and $s_{2}$ are generated using two arbitrary

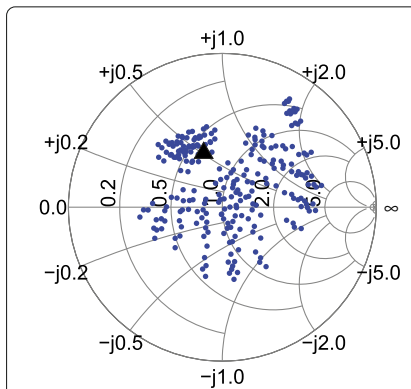

(a)

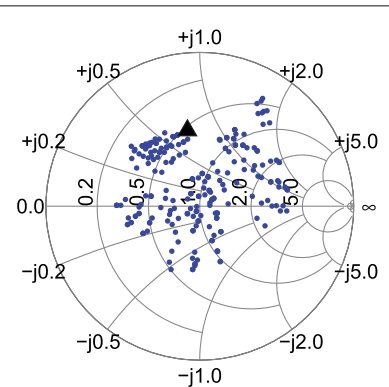

(b)

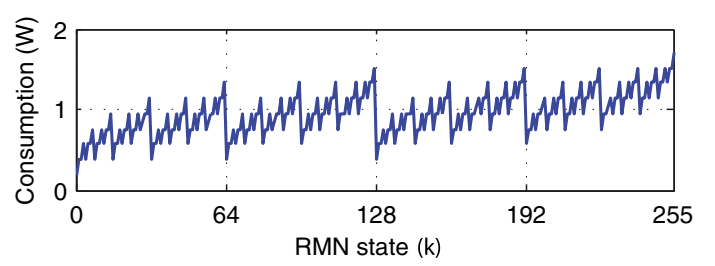

(c)

Fig. 6 Measured Smith chart coverage (blue points) and the best state (black triangle) with $29 \mathrm{dBm}$ power input. Path 1 (a) and path 2 (b) at $942 \mathrm{MHz}$. c The RMN consumption 
Table 2 Averaged drain efficiency and EVM measurements at different frequencies with a QPSK LTE signal (input power of $29 \mathrm{dBm}$ )

\begin{tabular}{|c|c|c|c|c|c|c|c|c|c|c|c|c|c|c|c|}
\hline \multirow[b]{2}{*}{$\begin{array}{l}\text { Freq } \\
(\mathrm{MHz})\end{array}$} & \multicolumn{7}{|l|}{ LINC } & \multicolumn{7}{|c|}{ LINC with RMN } & \multirow[b]{2}{*}{$\begin{array}{l}\triangle \text { eff } \\
(\%)\end{array}$} \\
\hline & $\begin{array}{l}\text { Pout }_{1} \\
\text { (W) }\end{array}$ & $\begin{array}{l}\mathrm{Pdc}_{1} \\
(\mathrm{~W})\end{array}$ & $\begin{array}{l}\text { Pout }_{2} \\
\text { (W) }\end{array}$ & $\begin{array}{l}\mathrm{Pdc}_{2} \\
(\mathrm{~W})\end{array}$ & $\begin{array}{l}\text { eff } \\
(\%)\end{array}$ & $\begin{array}{l}\text { EVM } \\
(\%)\end{array}$ & $\begin{array}{l}\text { ACLR } \\
\text { (dB) }\end{array}$ & $\begin{array}{l}\text { Pout }_{1} \\
\text { (W) }\end{array}$ & $\begin{array}{l}\mathrm{Pdc}_{1} \\
(\mathrm{~W})\end{array}$ & $\begin{array}{l}\text { Pout }_{2} \\
\text { (W) }\end{array}$ & $\begin{array}{l}\mathrm{Pdc}_{2} \\
(W)\end{array}$ & $\begin{array}{l}\text { eff } \\
(\%)\end{array}$ & $\begin{array}{l}\text { EVM } \\
(\%)\end{array}$ & $\begin{array}{l}\text { ACLR } \\
\text { (dB) }\end{array}$ & \\
\hline 927 & 5.57 & 17.82 & 5.61 & 17.98 & 31.23 & 2.33 & 54.57 & 6.15 & 14.43 & 6.12 & 14.36 & 42.63 & 1.96 & 56.07 & 36.50 \\
\hline 942 & 5.65 & 18.35 & 5.67 & 18.37 & 30.82 & 2.46 & 51.94 & 5.79 & 14.96 & 5.81 & 14.96 & 38.76 & 2.32 & 54.70 & 25.76 \\
\hline 958 & 5.76 & 18.69 & 5.75 & 18.72 & 30.81 & 2.36 & 53.22 & 5.90 & 15.56 & 5.90 & 15.67 & 37.82 & 2.03 & 56.72 & 22.75 \\
\hline
\end{tabular}

waveform generators (Agilent E4438C), which are synchronized and adjusted in gain and phase in order to ensure a free impairment setup. A LTE downlink signal (OFDM modulation with quadrature phase-shift keying (QPSK) subcarriers), with a bandwidth of $1.4 \mathrm{MHz}$ and a PAPR of $11.48 \mathrm{~dB}$ is decomposed into the LINC signals in order to carry out the experiments. Due to the bandwidth expansion suffered by the components $s_{1}$ and $s_{2}$ in a LINC architecture [13], these signals have a bandwidth larger than that of the original signal $s(t)$. The operating frequencies 927, 942, and $958 \mathrm{MHz}$ are within the band 8 (downlink) of the long-term evolution (LTE) standard. Two amplifiers are used as drivers (MiniCircuits ZHL-2-8). The devices under test (DUT) are two-class $\mathrm{AB}$ amplifiers based on a GaN HEMT transistor (CREE CGH40006P) with a gain of $15 \mathrm{~dB}$, which feed the RMNs inputs. The amplified signals are then combined by means of a $3 \mathrm{~dB}$ hybrid isolated combiner (MiniCircuits ZFSC2-2500), and the recomposed signals are captured with an oscilloscope (Infiniium DSO90804A). The RMN and the DUT consumption are provided by the power supply (Rohde-Schwarz HMC 8043). Figure 4b shows the DUTs, the RMN prototypes, and the combiner.

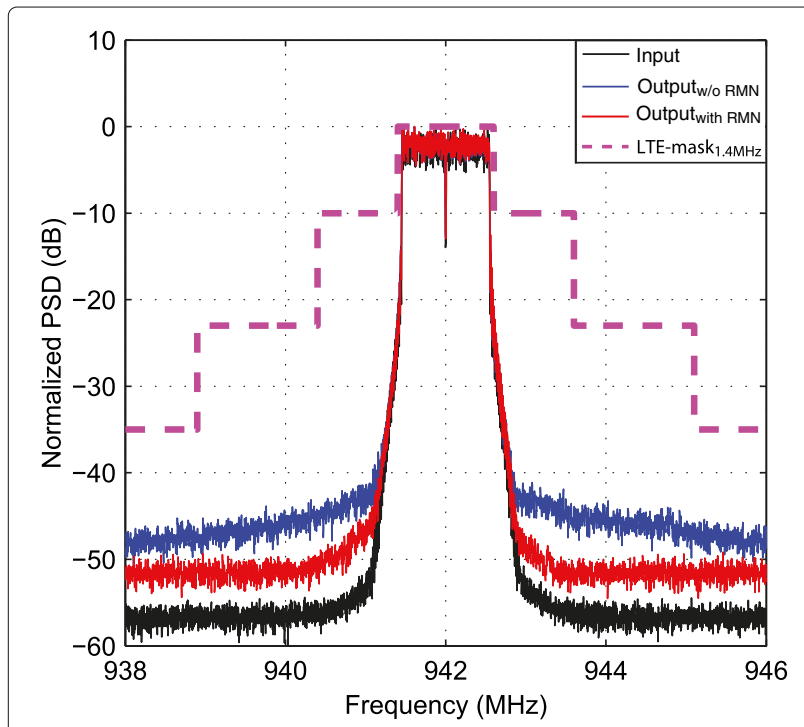

Fig. 7 Normalized power spectral density at $942 \mathrm{MHz}$ at the input (black), the output without (blue) and with RMN (red), and the LTE standard mask (pink) for 1.4 MHz bandwidth signal

\subsection{Optimization algorithm and measurements results}

Mathematically, the optimum of the following cost function, which is real-time evaluation and comes out from Eq. (15), is found by brute-force with a total time of about $2 \times N_{\text {states }} \times\left(t_{\text {on }}+t_{\text {proc }}\right)$, being the $t_{\text {on }}$ the time to switch on one PIN diode and $t_{\text {proc }}$ the time to process efficiency and update the maximum, provided gray state coding is used,

$J\left(k_{1}, k_{2}, P_{i n}\right)=\eta_{\mathrm{PA}+\mathrm{RMN}}^{(1)}\left(k_{1}, P_{i n}\right)+\eta_{\mathrm{PA}+\mathrm{RMN}}^{(2)}\left(k_{2}, P_{\text {in }}\right)$

where $P_{\text {in }}$ is the PA input power. The optimization process yields to a series of optimum states which fulfill

$$
\left\{\begin{array}{l}
k_{1}=\arg \max \eta_{P A}^{(1)}+R M N\left(k_{1}, P_{\text {in }}\right) \\
k_{2}=\arg \max \eta_{P A}^{(2)}+R M N
\end{array}\right.
$$

However, it can be surely improved applying methods which are already introduced in the literature [14, 15], where optimization techniques such as genetic algorithms or simplex methods are applied to RMNs in amplifier or antenna scenarios. In addition, it can also be possible to pre-calibrate the system in-house, for different operating
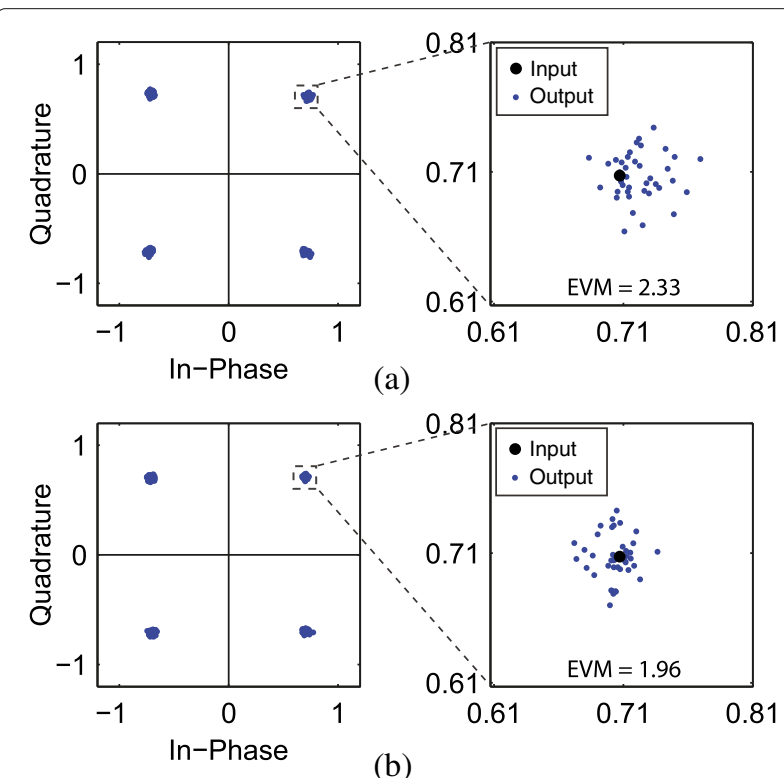

Fig. 8 Measured constellation diagram at $927 \mathrm{MHz}$ without the RMN (a) and with the RMN (b) 
conditions or frequency bands, and to save the optimum states in a LUT.

The comparison between $\eta_{\mathrm{PA}+\mathrm{RMN}}^{(1)}\left(k_{1}, P_{\text {in }}\right)$ and PA drain efficiency without the RMN (Fig. 3) allows to obtain a preliminary glimpse on a suitable input power $(29 \mathrm{dBm}$ in this work) in order to maximize efficiency with the RMN at a certain frequency. Despite theoretically both branches should be identical and consequently the RMN states, the final optimum results (Table 1) are different mainly because of the practical PA and RMN impairments (see Fig. 5).

In order to understand why these states maximize the cost function, coverage, consumption, and losses have been analyzed. RMN coverage at $942 \mathrm{MHz}$ with the state which offers the best drain efficiency and the RMN power consumption are plotted in Fig. 6 . The optimum state is not that which minimizes the RMN consumption because RF power is not maximized in such a case. Table 1 additionally presents not only ohmic losses $\left(L_{\Omega}\right)$ but also a set of transducer gain values which can be achieved for every path $\left(\mathrm{GT}_{1}\right.$ and $\left.\mathrm{GT}_{2}\right)$ [16]. The final estimated insertion losses $\left(\mathrm{IL}_{1}\right.$ and $\left.\mathrm{IL}_{2}\right)$ are set by the PA output load, but the ohmic losses are rather low. Anyway, the optimum state is a trade-off between losses and load pull, as the power consumption plays here an important role.

Table 2 presents a comparative overview between the classical LINC and the proposed RMN-LINC architecture, taking the RMNs consumption into account. As a figure-of-merit, the improvement in the drain efficiency is defined as

$$
\Delta \operatorname{eff}(\%)=100 \cdot \frac{\text { eff }_{\text {with_RMN }}-\text { eff }_{\text {without_RMN }}}{\text { eff }}
$$

Results show that it is possible to reach an enhancement with the RMNs in all cases, especially at $927 \mathrm{MHz}$ with an improvement of $36.50 \%$, reaching an averaged drain efficiency of $42.63 \%$.

The EVM (error vector magnitude) has been measured, and the adjacent channel leakage ratio (ACLR) has been estimated according to the standard to ensure the signal does not suffer distortions. Firstly, Fig. 7 shows the normalized power spectral density (NPSD) corresponding to the input and output signals, with and without the RMNs at $942 \mathrm{MHz}$. The transmitted waveforms meet the standard requirements, constrained by the $1.4 \mathrm{MHz}$ bandwidth LTE standard mask [17]. Finally, the constellation of the recomposed signals (Fig. 8) without (a) and with the RMN (b) at $927 \mathrm{MHz}$ shows the EVM has experimented an improvement (1.96\%).

\section{Conclusions}

In this paper, an enhanced classical LINC architecture based on RMNs has been presented proving successfully that their use can increase the transmitter efficiency. A novel analysis describes in terms of scattering parameters the effects of the RMNs in the LINC architecture. The comparison between both setups (with and without the RMNs) has been carried out in terms of the averaged drain efficiency at several frequencies, using a 1.4 MHz QPSK LTE signal. The RMN-LINC can provide not only better efficiency but also good linearity, as the ACLR values and the EVM analysis show.

\section{Abbreviations}

ACLR: Adjacent channel leakage ratio; DLM: Dynamic load modulation; DUT: Device under test; EVM: Error vector magnitude; FPGA: Field programmable gate array; LINC: Linear amplification with nonlinear components; NPSD: Normalized power spectral density; PA: Power amplifier; PAPR: Peak-to-average power ratio; PDF: Probability density function; RF: Radiofrequency; RMN: Reconfigurable matching network

\section{Funding}

This work was supported by the Spanish Government (Project TEC201458341-C4-2-R from MICINN and FEDER) and the Aragón Government and FSE (GCM T97) and a FPI grant from MICINN to the fifth author.

\section{Authors' contributions}

CM carried out the experimental measurements, result analysis, and article writing. PLC participated in the result analysis and article writing. PG-D took part in the article writing. JM, JRP-C, and CS-P did the reconfigurable matching network design and implementation. All authors read and approved the final manuscript.

\section{Competing interests}

The authors declare that they have no competing interests.

\section{Publisher's Note}

Springer Nature remains neutral with regard to jurisdictional claims in published maps and institutional affiliations.

Received: 2 January 2017 Accepted: 28 April 2017

Published online: 16 May 2017

\section{References}

1. PM Lavrador, TR Cunha, PM Cabral, JC Pedro, The linearity efficiency compromise. IEEE Microw. Mag., 44-58 (2010)

2. X Feng, W Yide, B Feuvrie, A Descamps, Y Ding, Z Yu, Analysis on LUT based digital predistortion using direct learning architecture for linearizing power amplifiers. EURASIP J. Wirel. Commun. and Netw. 2016(1), 1-14 (2016)

3. J Kim, B Fehri, S Boumaiza, J Wood, Power efficiency and linearity enhancement using optimized asymmetrical doherty power amplifiers. IEEE Trans. on Microw. Theory Tech. 59(2), 425-434 (2011)

4. CYu, A Zhu, A single envelope modulator-based envelope-tracking structure for multiple-input and multiple-output wireless transmitters. IEEE Trans. on Microw. Theory Tech. 60(10), 3317-3327 (2012)

5. P Garcia-Ducar, J de Mingo, PL Carro, A Valdovinos, Design and experimental evaluation of a LINC transmitter for OFDM systems. IEEE Trans. on Wireless Commun. 9(10), 2983-2987 (2010)

6. A Birafane, AB Kouki, On the linearity and efficiency of outphasing microwave amplifiers. IEEE Trans. on Microw. Theory Tech. 52(7), 1702-1708 (2004)

7. W Wu, S Cheng, Y Hsu, Maximum likelihood detection for coded combinerless LINC-OFDM systems. EURASIP J. Wirel. Commun. and Netw. 2016(1), 1-14 (2016)

8. HM Nemati, C Fager, U Gustavsson, R Jos, G Zirath, Design of varactor-based tuneable matching networks for dynamic load modulation of high power amplifiers. IEEE Trans. on Microw. Theory Tech. 57(5), 1110-1118 (2009)

9. AM Mahmoud, S Boumaiza, RR Mansour, in Proc. of the 40 th Europ. Microw. Conf. Novel reconfigurable fundamental/harmonic matching network for enhancing the efficiency of power amplifiers, (2010), pp. 1122-1125 
10. FH Raab, Efficiency of outphasing RF power-amplifier systems. IEEE Trans. on Commun. COM-33(10), 1094-1099 (1985)

11. JC Pedro, LC Nunes, PM Cabral, A simple method to estimate the output power and efficiency load-pull contours of class-B power amplifiers. IEEE Trans. on Microw. Theory Tech. 63(4), 1239-1249 (2015)

12. C Sanchez-Perez, J de Mingo, PL Carro, P Garcia-Ducar, Design and applications of a 300-800 MHz tuneable matching network. IEEE J. Emerg. Sel. Top. Circ. Syst. 3(4), 531-540 (2013)

13. AK Mustafa, S Ahmed, M Faulkner, Bandwidth limitation for the constant envelope components of an OFDM signal in a LINC architecture. IEEE Trans. on Circuits and Syst. I: Reg. Papers. 60(9), 2502-2510 (2013)

14. D Qiao, R Molfino, SM Lardizabal, B Pillans, PM Asbeck, G Jerinic, An intelligently controlled RF power amplifier with a reconfigurable MEMS-varactor tuner. IEEE Trans. on Microw. Theory Tech. 53(3), 1089-1095 (2005)

15. J de Mingo, A Valdovinos, A Crespo, D Navarro, P Garcia, An RF electronically controlled impedance tuning network design and its application to an antenna input impedance automatic matching system. IEEE Trans. on Microw. Theory Tech. 52(2), 489-497 (2004)

16. DM Pozar, Microwave Amplifier Design, 2nd Ed., Microwave Engineering, (1998), pp. 558-562

17. 3GPP TSGRAN: User equipment (UE) radio transmission and reception (FDD). Technical Specification ETSI TS136.104 V13.3.0, (2016), http://www. etsi.org/standards-search

\section{Submit your manuscript to a SpringerOpen ${ }^{\circ}$ journal and benefit from:}

- Convenient online submission

- Rigorous peer review

- Immediate publication on acceptance

- Open access: articles freely available online

- High visibility within the field

- Retaining the copyright to your article

Submit your next manuscript at $\gg$ springeropen.com 